\title{
Multi-bandwidth image reconstruction in photoacoustic tomography
}

Cheng-Ying Chou, Mark A. Anastasio, Jin Zhang, Geng Ku, Xinmai Yang, et al.

Cheng-Ying Chou, Mark A. Anastasio, Jin Zhang, Geng Ku, Xinmai Yang, Lihong V. Wang, "Multi-bandwidth image reconstruction in photoacoustic tomography," Proc. SPIE 6856, Photons Plus Ultrasound: Imaging and Sensing 2008: The Ninth Conference on Biomedical Thermoacoustics, Optoacoustics, and Acousto-optics, 68561G (28 February 2008); doi: 10.1117/12.764127

SPIE. Event: SPIE BiOS, 2008, San Jose, California, United States 


\title{
Multi-bandwidth image reconstruction in photoacoustic tomography
}

\author{
Cheng-Ying Chou ${ }^{a}$, Mark A. Anastasio ${ }^{b}$, Jin Zhang ${ }^{b}$, Geng $\mathrm{Ku}^{c}$, Xinmai Yang ${ }^{c}$, \\ and Lihong V. Wang ${ }^{c}$ \\ ${ }^{a}$ Department of Bio-Industrial Mechatronics Engineering \\ National Taiwan University, Taipei, Taiwan 106 \\ ${ }^{b}$ Medical Imaging Research Center, Department of Biomedical Engineering \\ Illinois Institute of Technology, Chicago, IL 60616 \\ ${ }^{c}$ Optical Imaging Laboratory, Department of Biomedical Engineering \\ Washington University in St. Louis, St. Louis, MO 63130
}

\begin{abstract}
Photoacoustic tomography (PAT), also referred to optoacoustic tomography, is a hybrid imaging technique that combines an optical contrast mechanism and ultrasonic detection principles. The laser-induced photoacoustic signals in PAT are broadband in nature, but only a bandpass approximation of the signal is recorded by use of a conventional ultrasonic transducers due to its limited bandwidth. To circumvent this, a PAT system has been developed that records photoacoustic signals by use of multiple ultrasonic transducers that possess different central frequencies. In this work, we investigate a sensor fusion methodology for combining the multiple measurements to obtain an estimate of the true photoacoustic signal that is superior to that obtainable by use of any single transducer measurement. From the estimated photoacoustic signals, three-dimensional images of the optical absorption distribution are reconstructed and are found to possess improved accuracy and statistical properties as compared to the single transducer case. Preliminary computer-simulation studies are presented to demonstrate and investigate the proposed method.
\end{abstract}

Keywords: photoacoustic tomography, sensor fusion

\section{INTRODUCTION}

Photoacoustic tomography (PAT $)^{1-4}$ is a rapidly emerging bioimaging method that exploits an electromagnetic contrast mechanism and ultrasound detection principles. It has demonstrated great promise for important applications including functional brain imaging of animals ${ }^{5-7}$ and soft-tissue characterization..$^{8,9}$ In PAT, a short laser pulse is used to irradiate a biological tissue, as depicted in Fig. 1. Under conditions of thermal confinement, ${ }^{10}$ the thermoacoustic effect results in the generation of pressure wavefields, i.e., photoacoustic signals, that can be recorded by ultrasonic transducers whose locations span a measurement aperture. The goal of PAT is to reconstruct an image depicting of the spatially variant optical absorption distribution in the tissue from knowledge of the measured photoacoustic signals. Assuming idealized imaging conditions and ultrasonic transducers that are wide-band and point-like, a variety of reconstruction algorithms ${ }^{11-14}$ have been developed in recent years to accomplish this.

The laser-induced photoacoustic signals in PAT are broadband in nature, but only a bandpass approximation of the signal is recorded by use of a conventional ultrasonic transducers due to its limited temporal frequency bandwidth. An image reconstructed from such data will be bandpass filtered ${ }^{15,16}$ and, in general, can only be interpreted qualitatively. To circumvent this, a PAT system has been developed ${ }^{17}$ that records photoacoustic signals by use of multiple ultrasonic transducers that possess different central frequencies. Through experimental studies, it was demonstrated that the use of higher frequency transducers yielded images with higher spatial resolution, while the use of lower frequency transducers yielded images possessing higher signal-to-noise ratios (SNRs). These results suggest that it is highly desirable to reconstruct an image from an appropriate combination

Further author information: Send correspondence to Mark A. Anastasio, Ph.D.: E-mail: anastasio@iit.edu

Photons Plus Ultrasound: Imaging and Sensing 2008: The Ninth Conference on Biomedical

Thermoacoustics, Optoacoustics, and Acousto-optics, edited by Alexander A. Oraevsky, Lihong V. Wang,

Proc. of SPIE Vol. 6856, 68561G, (2008) · 1605-7422/08/\$18 - doi: 10.1117/12.764127

Proc. of SPIE Vol. 6856 68561G-1 
of all the available transducer measurements, which would yield an image with high spatial resolution and high SNR.

In this work, we investigate a sensor fusion methodology for combining measurements from multiple transducers to obtain an estimate of the true photoacoustic signal that is superior to that obtainable by use of any single transducer measurement. This is accomplished via a Fourier transform-based multi-channel deconvolution framework employing a statistically motivated combination coefficient. From the estimated photoacoustic signals, three-dimensional (3D) images of the optical absorption distribution are reconstructed and are found to possess improved accuracy and statistical properties as compared to the single transducer case. Preliminary computer-simulation studies are presented to demonstrate and investigate the proposed method.

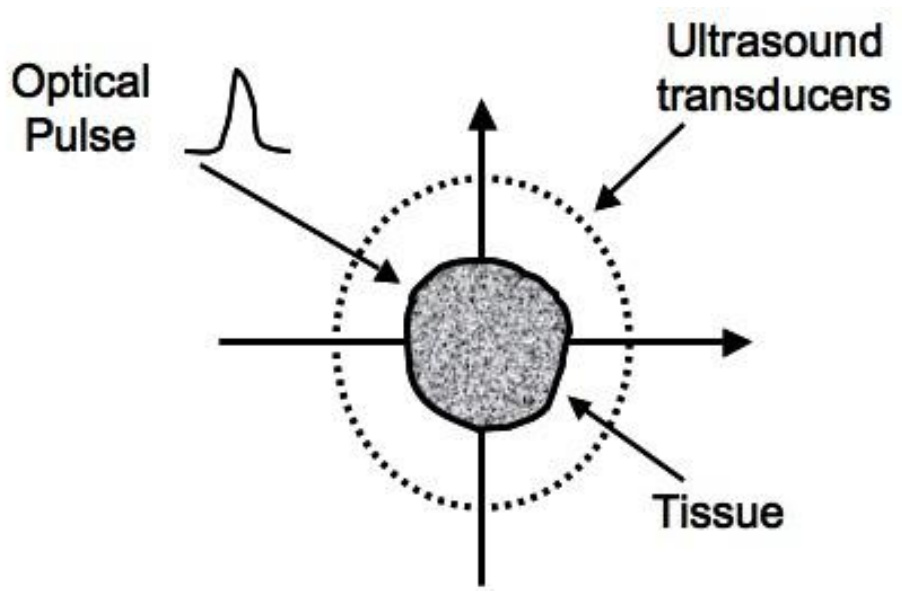

Figure 1. A schematic of the PAT imaging experiment.

\section{PAT IMAGING MODEL}

We consider that a laser pulse with Dirac delta time-dependence $\delta(t)$ irradiates a tissue sample residing completely inside a spherical measurement aperture $\Omega_{0}$ of radius $R_{0}$. The resulting pressure wavefield $p\left(\vec{r}_{0}, t\right)$, representing the photoacoustic signal, at transducer location $\vec{r}_{0} \in \Omega_{0}$ is related to the optical absorption distribution $A(\vec{r})$ as

$$
p^{\text {ideal }}\left(\vec{r}_{0}, t\right)=\eta \int_{V} d \vec{r} A(\vec{r}) \frac{d}{d t} \frac{\delta\left(t-\frac{\left|\vec{r}_{0}-\vec{r}\right|}{c_{0}}\right)}{4 \pi\left|\vec{r}_{0}-\vec{r}\right|},
$$

where $c_{0}$ is the speed-of-sound in the object and homogeneous background medium, and $\eta$ is a known constant. The superscript 'ideal' denotes that the photoacoustic signal has not been degraded by the response of the transducer.

With consideration of a finite detector bandwidth, finite laser pulse duration, and measurement noise, the measured photoacoustic signals are related to the ideal ones by

$$
p^{\text {meas }}\left(\vec{r}_{0}, t\right)=\left[p^{\text {ideal }}\left(\vec{r}_{0}, t\right) \star h(t)\right]+n\left(\vec{r}_{0}, t\right),
$$

where $h(t)$ is the impulse response that describes the finite detector bandwidth and laser pulse duration, $n\left(\vec{r}_{0}, t\right)$ denotes measurement noise, and $\star$ indicates a one-dimensional (1D) convolution. The real-world reconstruction problem of PAT can be interpreted as two steps. Firstly, an estimate of the ideal photoacoustic data $p^{\text {ideal }}\left(\vec{r}_{0}, t\right)$ must be obtained from knowledge of the measured data $p^{\text {meas }}\left(\vec{r}_{0}, t\right)$. This represents a $1 \mathrm{D}$ deconvolution problem that is ill-posed and difficult to solve accurately. To avoid a large noise amplification, only a bandpass approximation of $p^{\text {ideal }}\left(\vec{r}_{0}, t\right)$ can be reconstructed. Secondly, from knowledge of the estimate of $p^{\text {ideal }}\left(\vec{r}_{0}, t\right)$, an image depicting $A(\vec{r})$ is obtained by inverting Eqn. (1) by use of a tomographic reconstruction algorithm. As described in the following section, an appropriate use of measurements from transducers with different temporal responses can mitigate the deconvolution problem and therefore yield improved reconstructed images. 


\section{MULTI-BANDWIDTH IMAGE RECONSTRUCTION}

Consider the case where at each measurement location $\vec{r}_{0}$ the photoacoustic signals are recorded by use of $M$ ultrasonic transducers having distinct temporal responses. The temporal responses are described by the impulse response functions $h_{m}(t)$ and the corresponding measurement data are denoted by $p_{m}\left(\vec{r}_{0}, t\right)$, where $m=1,2, \cdots, M$ is the transducer index. The measurement model in Eqn. (2) indicates that

$$
p_{m}\left(\vec{r}_{0}, t\right)=\left[p^{\text {ideal }}\left(\vec{r}_{0}, t\right) \star h_{m}(t)\right]+n\left(\vec{r}_{0}, t\right), \quad m=1,2, \cdots, M .
$$

We seek to obtain an estimate of $p^{\text {ideal }}\left(\vec{r}_{0}, t\right)$ from knowledge of the $M$ transducer measurements $p_{m}\left(\vec{r}_{0}, t\right)$. This is a classic multi-channel restoration problem, and we propose to solve it by use of the Fourier-space weighting method described below.

Let $P_{m}\left(\vec{r}_{0}, \omega\right)$ denote the $1 \mathrm{D}$ temporal Fourier transform of the measured signal $p_{m}(t)$ :

$$
P_{m}\left(\vec{r}_{0}, \omega\right)=\int_{-\infty}^{\infty} d t p_{m}\left(\vec{r}_{0}, t\right) \exp (-j \omega t)
$$

Similarly, let $P^{\text {ideal }}\left(\vec{r}_{0}, \omega\right)$ and $H_{m}(\omega)$ denote the 1D temporal Fourier transforms of $p^{\text {ideal }}\left(\vec{r}_{0}, t\right)$ and $h_{m}(t)$, respectively. For each transducer, an estimate of $P^{i d e a l}\left(\vec{r}_{0}, \omega\right)$ can be obtained as

$$
P_{m}^{\text {ideal }}\left(\vec{r}_{0}, \omega\right) \approx \frac{P_{m}\left(\vec{r}_{0}, \omega\right)}{H_{m}(\omega)}
$$

where the subscript ' $m$ ' has been added to $P^{\text {ideal }}\left(\vec{r}_{0}, \omega\right)$ to denote that it was estimated from knowledge of $P_{m}\left(\vec{r}_{0}, \omega\right)$ and $H_{m}(\omega)$. Accordingly, if measurements are available from $M$ transducers, we have $M$ estimates $P_{m}^{\text {ideal }}\left(\vec{r}_{0}, \omega\right)$ of the true $P^{\text {ideal }}\left(\vec{r}_{0}, \omega\right)$. In the presence of data noise, these estimates will generally be distinct. Of course, some of these estimates will have severely amplified noise levels for frequencies at which $H_{m}(\omega)$ takes on small values.

A simple way to mitigate this fact, is to combine the available estimates, in a statistically motivated way, to form a final estimate of $P^{\text {ideal }}\left(\vec{r}_{0}, \omega\right)$ as

$$
P^{f i n a l}\left(\vec{r}_{0}, \omega\right)=\sum_{m=1}^{M} W_{m}(\omega) P_{m}^{\text {ideal }}\left(\vec{r}_{0}, \omega\right)=\sum_{m=1}^{M} W_{m}(\omega) \frac{P_{m}\left(\vec{r}_{0}, \omega\right)}{H_{m}(\omega)},
$$

where $W_{m}(\omega)$ are frequency-dependent combination coefficients that are generally complex-valued. If the secondorder statistical properties of $P_{m}\left(\vec{r}_{0}, \omega\right)$ are known, the combination coefficients $W_{m}(\omega)$ can be designed to minimize the variance of $P^{\text {final }}\left(\vec{r}_{0}, \omega\right)$ at all frequencies $\omega$. However, even if such information is not available, effective choices for $W_{m}(\omega)$ can still be identified.

According to Eqn. (5), the variance of $P_{m}^{\text {ideal }}\left(\vec{r}_{0}, \omega\right)$ behaves as

$$
\operatorname{Var}\left\{P_{m}^{\text {ideal }}\left(\vec{r}_{0}, \omega\right)\right\} \propto \frac{1}{\left|H_{m}(\omega)\right|^{2}} .
$$

The combination coefficient that weights the $m$-th estimate should have an $\omega$-dependence that is inversely proportional to Eqn. (7), to avoid a large noise amplification in the final estimate. The combination coefficients should also satisfy

$$
\sum_{m=1}^{M} W_{m}(\omega)=1
$$

to ensure that $P^{\text {final }}\left(\vec{r}_{0}, \omega\right)$ is an unbiased estimate of $P^{\text {ideal }}\left(\vec{r}_{0}, \omega\right)$. Based on these requirements, the $W_{m}(\omega)$ can be chosen as

$$
W_{m}(\omega)=\frac{\left|H_{m}(\omega)\right|^{2}}{\sum_{k=1}^{M}\left|H_{k}(\omega)\right|^{2}},
$$


and the estimator in Eqn. (6) takes the explicit form

$$
P^{\text {final }}\left(\vec{r}_{0}, \omega\right)=\sum_{m=1}^{M} P_{m}\left(\vec{r}_{0}, \omega\right) \frac{H_{m}^{*}(\omega)}{\sum_{k=1}^{M}\left|H_{k}(\omega)\right|^{2}},
$$

where $*$ denotes complex conjugation. Note that if $H_{m}(\omega)=0$ for some frequency $\omega$, the corresponding combination coefficient $W_{m}(\omega)=0$, ensuring that $P_{m}\left(\vec{r}_{0}, \omega\right)$ will not contribute to the final estimate at that frequency. Similarly, if $H_{m}(\omega)$ takes on a relatively small value, the corresponding $W_{m}(\omega)$ will be small. The opposite is true if $H_{m}(\omega)$ is relatively large. Application of the inverse Fourier transform to $P^{f i n a l}\left(\vec{r}_{0}, \omega\right)$ yields the final estimate of pressure wavefield from which the optical absorption distribution of the object can be reconstructed.

\section{NUMERICAL STUDIES AND RESULTS}

Computer-simulation studies were conducted to demonstrate and investigate the proposed multi-bandwidth image reconstruction method.

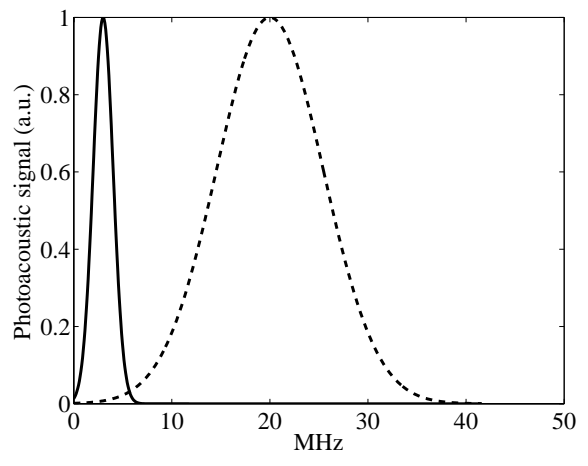

(a)

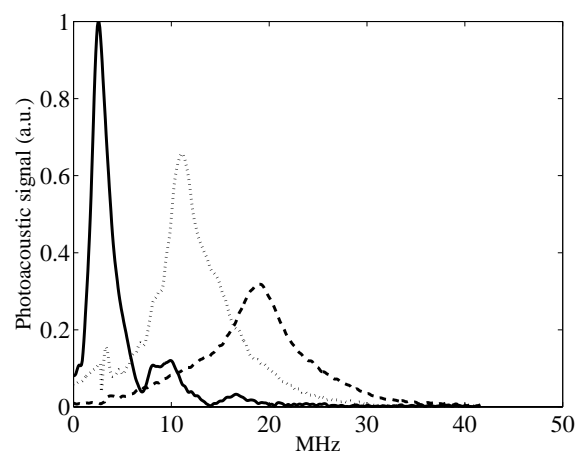

(b)

Figure 2. The transducer transfer functions for (a) System A and (b) System B.

\subsection{Computer-Simulations}

Mathematical phantom and simulation data: A 3D numerical phantom was employed to represent the optical absorption distribution $A(\vec{r})$. The phantom was comprised of 12 spheres possessing different optical absorption values, with the radius of the largest sphere set at $1.0 \mathrm{~cm}$. The measurement aperture corresponded to a sphere of $5 \mathrm{~cm}$ that enclosed the object. Along the vertical direction, 129 uniformly spaced planes that intersected the scanning aperture were chosen. The intersection of each plane with the aperture defined a circle, over which 360 point-like transducers were assumed to be uniformly distributed. Under the assumption that the object was acoustically homogeneous, idealized photoacoustic pressure signals were generated at each transducer location on the measurement sphere.

From knowledge of the ideal measurement data, two multi-bandwidth PAT imaging systems were simulated. In the first system, referred to as 'System A', two different transducers were assumed to record the photoacoustic signals at each transducer location. The transfer functions of the transducers are shown in Fig. 2-(a), and have the forms of truncated Gaussian functions with central frequencies of $3 \mathrm{MHz}$ and $20 \mathrm{MHz}$. The second system, referred to as 'System B', employed three different transducers to record the photoacoustic signals. The transfer functions were modeled after the experimental measurements and are depicted in Fig. 2-(b). The central frequencies were $3.5 \mathrm{MHz}, 10 \mathrm{MHz}$ and $20 \mathrm{MHz}$. To produce simulated measurement data for both systems, the ideal photoacoustic signals were degraded by use of the transducer transfer functions and random measurement noise was introduced. The measurement noise was sampled from a Gaussian distribution whose standard deviation $\sigma$ was approximately $10 \%$ of the peak noiseless value. 
Image reconstruction and evaluation: At each transducer location, the temporal Fourier transforms of the available measurements were computed and combined by use of Eqn. (10) (with $M=2$ for System A and $M=3$ for System B) to form a final Fourier estimate of the photoacoustic signal. Subsequently, the universal backprojection algorithm ${ }^{12}$ was employed to reconstruct an estimate of $A(\vec{r})$. In order to investigate the statistical properties of the reconstructed images, ensembles containing 600 noisy realizations of each transducer measurement were computed, from which 600 noisy estimates of $A(\vec{r})$ were reconstructed as described above. Empirical estimates of the image variance and SNR were computed.

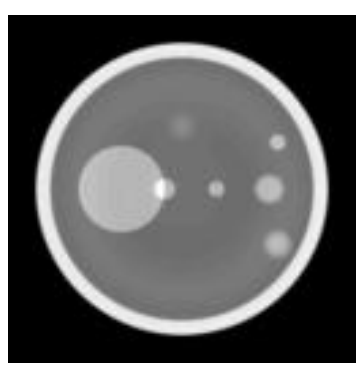

(a) $A_{\text {ideal }}$

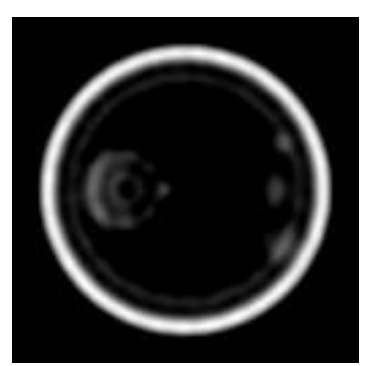

(b) $A_{3}$

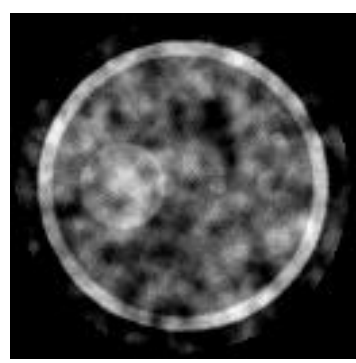

(c) $A_{20}$

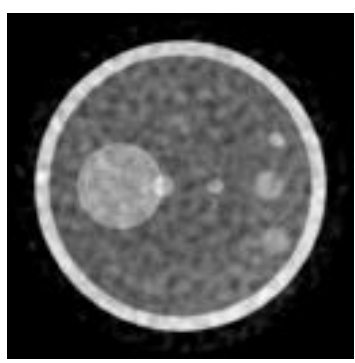

(d) A Aptimal

Figure 3. Images of $A(\vec{r})$ for System A reconstructed from (a) ideal pressure data, (b) $3 \mathrm{MHz}$ transducer data, (c) 20 $\mathrm{MHz}$ transducer data, and (d) the multi-bandwidth reconstruction.

\subsection{Numerical results}

Reconstructed images for System A are contained in Fig. 3. The reconstructed slice is through the central $(z=0)$ plane of the object, and the image reconstructed from the noiseless ideal photoacoustic signals is shown in Fig. 3-(a). Images reconstructed from the noisy data corresponding to the $3 \mathrm{MHz}$ and $20 \mathrm{MHz}$ transducers are shown in Fig. 3-(b) and (c). Each of these single-bandwidth images was computed by deconvolving the measured signal with respect to the corresponding impulse response [see Eqn. (2)], followed by application of the universal backprojection algorithm. As expected, due to the ill-posed nature of the deconvolution resulting from the limited transducer bandwidth, both of the single-channel images are highly contaminated by noise. The multi-bandwidth reconstructed image is shown in Fig. 3-(d), and possess superior spatial resolution and lower noise levels than either of the single-bandwidth reconstructions.

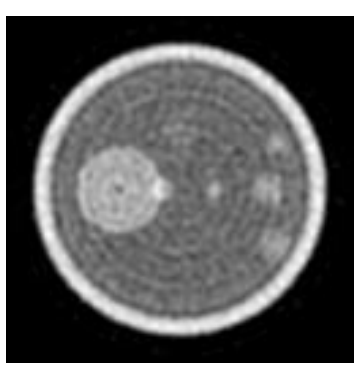

(a) $A_{3.5}$

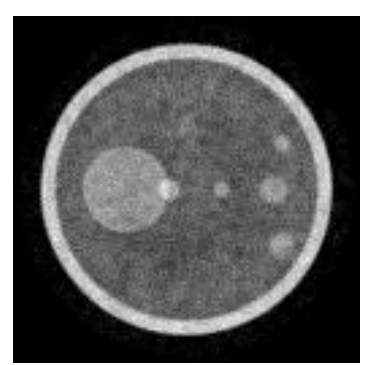

(b) $A_{10}$

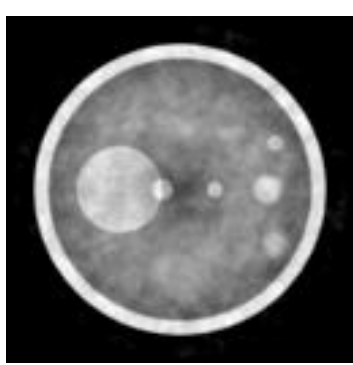

(c) $A_{20}$

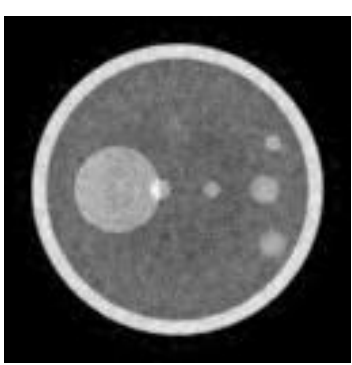

(d) A Aptimal

Figure 4. Images of $A(\vec{r})$ for System B reconstructed from (a) $3.5 \mathrm{MHz}$ transducer data, (b) $10 \mathrm{MHz}$ transducer data, (c) $20 \mathrm{MHz}$ transducer data, and (d) the multi-bandwidth reconstruction. 
Reconstructed images for System B are contained in Fig. 4. Figures 4-(a)-(c) display single-bandwidth reconstructions corresponding to $3.5 \mathrm{MHz}, 10 \mathrm{MHz}$, and $20 \mathrm{MHz}$ transducers, respectively. The multi-bandwidth image is displayed in Fig. 4-(d). As expected, the multi-bandwidth reconstruction appears to be the most accurate and least affected by noise. It should be noted that in our simulation studies, the single bandwidth results are not as terrible as one might expect. This can be attributed to as least two reasons. Firstly, the noise level is modest. As the noise level is increased, the advantages of the multi-bandwidth reconstruction will become more prominent. Secondly, the higher-frequency components of the photoacoustic signal play a more important role in the reconstruction process than do the lower-frequency ones, and all of the transfer functions 'capture' a wide range of frequency components that are important to accurately describe true photoacoustic signal.

The SNR images, computed from the ensemble of 600 noisy estimates of $A(\vec{r})$ are contained in Fig. 5. The SNR images for the single-channel $3.5 \mathrm{MHz}$ and $20 \mathrm{MHz}$ reconstructions are displayed in Figs. 5-(a) and (b). Although not shown, the SNR image for the $10 \mathrm{MHz}$ transducer case has values that lie between these two. The SNR image corresponding to the multi-bandwidth reconstruction is displayed in Fig. 5-(c). As confirmed by profiles through the SNR maps shown in Fig. 6, the SNR of the multi-bandwidth image is higher than the single-bandwidth reconstructions at almost all spatial positions.

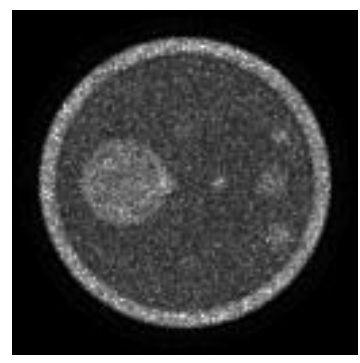

(a) $\mathrm{SNR}_{3.5}$

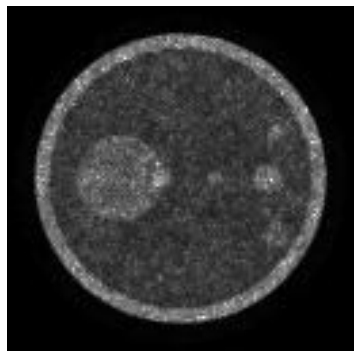

(b) $\mathrm{SNR}_{20}$

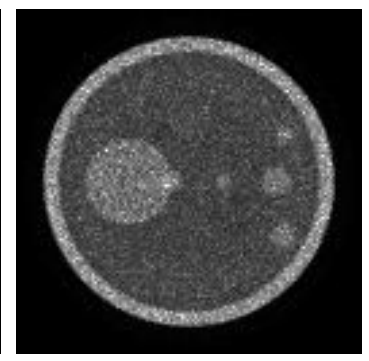

(c) $\mathrm{SNR}_{\text {optimal }}$

Figure 5. Computed SNR maps of images corresponding to (a) $3.5 \mathrm{MHz}$ transducer data, (b) $20 \mathrm{MHz}$ transducer data, (c) multi-bandwidth image.

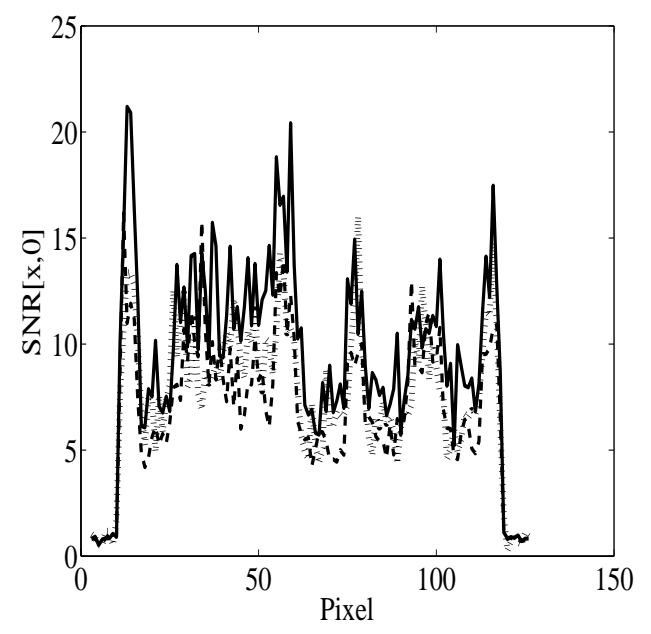

Figure 6. Profiles though the SNR maps shown in Fig. 5. Solid, dashed and dotted curves correspond to the multibandwidth, $3.5 \mathrm{MHz}$ and $20 \mathrm{MHz}$ cases, respectively. 


\section{SUMMARY}

We have proposed and investigated a sensor fusion method for multi-bandwidth PAT. The method combines photoacoustic signal measurements from multiple transducers to obtain a significantly improved estimate of the true photoacoustic signal. This was accomplished via a Fourier transform-based multi-channel deconvolution framework employing a statistically motivated combination coefficient. Computer-simulation studies established the effectiveness of the method. From the estimated photoacoustic signals, 3D images of the optical absorption distribution were reconstructed and found to possess improved accuracy and statistical properties as compared to the single transducer case.

There remain several important topics for future investigations. When acquiring measurements with highfrequency transducers, the affects of acoustic attenuation may be significant, and should be incorporated into the method. ${ }^{18}$ Additionally, the model can be generalized to compensate for the effects of anisotropic detection sensitivity of the transducers. Finally, the method should be systematically evaluated by use of well-calibrated experimental data and modified as necessary to ensure robust performance.

\section{REFERENCES}

1. M. Xu and L. V. Wang, "Biomedical photoacoustics," Review of Scientific Instruments 77(041101), 2006.

2. L. V. Wang, "Ultrasound-mediated biophotonic imaging: A review of acousto-optical tomography and photo-acoustic tomography," Disease Markers 19, pp. 123-138, 2003-2004.

3. R. A. Kruger, P. Liu, R. Fang, and C. Appledorn, "Photoacoustic ultrasound (PAUS) reconstruction tomography," Medical Physics 22, pp. 1605-1609, 1995.

4. A. A. Oraevsky and A. A. Karabutov, Optoacoustic Tomography, CRC Press LLC, 2003.

5. X. Wang, G. Ku, M. Wegiel, D. Bornhop, G. Stoica, and L. V. Wang, "Noninvasive photoacoustic angiography of animal brains in vivo with near-infrared light and an optical contrast agent," Optics Letters 29(7), pp. 730-733, 2004.

6. X. Wang, Y. Pang, G. Ku, X. Xie, G. Stoica, and L. V. Wang, "Non-invasive laser-induced photo-acoustic tomography of the brain in vivo: structural and functional neuroimaging," Nature Biotechnology 21, p. 803, 2003.

7. R. A. Kruger, W. L. Kiser, D. R. Reinecke, G. A. Kruger, and K. D. Miller, "Thermoacoustic optical molecular imaging of small animals," Molecular Imaging 2, pp. 113-123, 2003.

8. X. Wang, Y. Xu, M. Xu, S. Yokoo, E. Fry, and L. V. Wang, "Photo-acoustic tomography of biological tissues with high cross-section resolution: Reconstruction and experiment," Medical Physics 29, pp. 2799$2805,2002$.

9. G. Ku, B. D. Fornage, X. Jin, M. Xu, K. K. Hunt, and L. V. Wang, "Thermoacoustic and photoacoustic tomography of thick biological tissues toward breast imaging," Technology in Cancer Research and Treatment 4, pp. 559-566, 2005.

10. A. C. Tam, "Application of photo-acoustic sensing techniques," Rev. Mod. Phys. 58, pp. 381-431, 1986.

11. D. Finch, S. Patch, and Rakesh, "Determining a function from its mean values over a family of spheres," SIAM Journal of Mathematical Analysis 35, pp. 1213-1240, 2004.

12. Y. Xu and L. V. Wang, "Universal back-projection algorithm for photoacoustic computed tomography," Physical Review E 71(016706), 2005.

13. L. A. Kunyansky, "Explicit inversion formulae for the spherical mean radon transform," Inverse Problems 23, pp. 373-383, 2007.

14. M. A. Anastasio, J. Zhang, X. Pan, Y. Zou, G. Keng, and L. V. Wang, "Half-time image reconstruction in thermoacoustic tomography," IEEE Transactions on Medical Imaging 24, pp. 199-210, 2005.

15. M. Xu and L. V. Wang, "Analytic explanation of spatial resolution related to bandwidth and detector aperture size in thermoacoustic or photoacoustic reconstruction," Physical Review E 67(056605), 2003.

16. M. A. Anastasio, J. Zhang, D. Modgil, and P. La Riviere, "Application of inverse source concepts to photoacoustic tomography," Inverse Problems 23, pp. S21-S35, 2007.

17. G. Ku, X. Wang, G. Stoica, and L. V. Wang, "Multiple-bandwidth photoacoustic tomography," Physics in Medicine and Biology 49, pp. 1329-1338, 2004.

18. P. L. Riviere, J. Zhang, and M. A. Anastasio, "Image reconstruction in optoacoustic tomography for dispersive acoustic media," Optics Letters 31, pp. 781-783, 2006. 